\title{
HPLC-ESI-MS/MS Profiling of Phenolic Acids, Flavonoids And Sesquiterpene Lactones from Xanthium spinosum
}

\author{
ERZSEBET VARGA ${ }^{1}$, ERZSEBET DOMOKOS ${ }^{*}$, HAJNAL KELEMEN ${ }^{3}$, IBOLYA FULOP ${ }^{4}$, \\ LASZLO KURSINSZKI ${ }^{5}$ \\ ${ }^{1}$ University of Medicine, Pharmacy, Science and Technology "George Emil Palade" of Targu Mures, Department of \\ Pharmacognosy and Phytotherapy, 38 Gheorghe Marinescu Str., 540139, Mures, Romania \\ ${ }^{2}$ University of Medicine, Pharmacy, Science and Technology "George Emil Palade" of Targu Mures, Department of \\ Pharmaceutical Botany, 38 Gheorghe Marinescu Str., 540139, Mures, Romania \\ ${ }^{3}$ University of Medicine, Pharmacy, Science and Technology "George Emil Palade" of Targu Mures, Department of \\ Pharmaceutical Chemistry, 38 Gheorghe Marinescu Str., 540139, Mures, Romania \\ ${ }^{4}$ University of Medicine, Pharmacy, Science and Technology "George Emil Palade" of Targu Mures, Faculty of Pharmacy, \\ Department of Toxicology and Biopharmacy, 38 Gheorghe Marinescu Str., 540139, Mures,Romania \\ ${ }^{5}$ Department of Pharmacognosy, Semmelweis University, Budapest, Ulloi 26, Hungary
}

Abstract. Bathurst burr (Xanthium spinosum L.) is used worldwide in traditional medicine to treat a diverse range of health problems including urinary problems associated with various prostate diseases. The aim of this study was to complete the identification and structural characterization of the chemical constituents from the aerial part of X. spinosum by high-performance liquid chromatography coupled with electrospray ionization tandem mass spectrometry. From the unequivocally detected and characterized compounds of aqueous-methanol extract, protocatechuic acid, 4-O-caffeoylquinic acid and 3,5-di-O-caffeoylquinic acid were found for the first time in X. spinosum. Besides these compounds, 25 phenolics (including 7 hydroxybenzoic derivatives, 13 hydroxycinnamic derivatives, one benzyl alcohol-hexose-pentose, and 4 flavonoids), 6 sesquiterpenes and 3 diterpenes were tentatively identified.

Keywords: Xanthium spinosum, protocatechuic acid; 4-O-caffeoylquinic acid; 3,5-di-O-caffeoylquinic acid; HPLC-ESI-MS/MS

\section{Introduction}

Bathurst burr (Xanthium spinosum L.), from the family Asteraceae is an annual herb originated from South America. The herb is used traditionally in Romania for urinary problems and various prostate diseases [1]. The pharmacological profile of $X$. spinosum includes the anti-inflammatory activity attributed to its flavonoids. The sesquiterpene lactone xanthatin from the leaves has antibacterial and antifungal properties [2,3]. Xanthatin extracted from the aerial parts of the plant shows inhibitory activity against a wide variety of viruses. Also, it was revealed that xanthatin has a strong anti-angiogenesis capacity in vitro [4]. The infusion and tincture obtained from $X$. spinosum aerial parts are efficient in the treatment of rats with induced benign prostate hypertrophia $[5,6]$.

The revealed chemical constituents of $X$. spinosum were classified into three main groups of active principles [7]: phenolics (caffeic acid, chlorogenic acid, quercetin, pendulin, jacein, centaurein, and patuletin-3-O-glycoside), sesquiterpenes (2-acetoxi-4- $O$ - hydroxideacetylxanthanol, $1 \alpha, 5 \alpha$-epoxy-1,5dihydroxanthatin, 1 $\beta, 5 \beta$-epoxy-1,5-dihydroxanthatin, desacetyl xanthiuminol, 2-hydroxi-4-O-acetoxideacetylxanthanol, 2-hydroxi-4-oxo-deacetylxanthanol, 2- $O$-acetyl-4-oxo-desacetylxanthanol, 2-oxo4- $O$-acetyldesacetylxanthanol, xanthatin, xanthinin, 2-desacetyl-8-epi-xanthumanol-4- $O$ - $\beta$-D-galactopyranoside, xanthumin, deacetilxanthumin, stizolicin and solstitialin), and diterpenes

\footnotetext{
*email: erzsebet.domokos@umfst.ro,domokosrzsbet@gmail.com
} 
(3', 4'-didesulphated-carboxyatractyloside, 3', 4'-didesulphated-atractyloside, 4'-desulphated- carboxy-

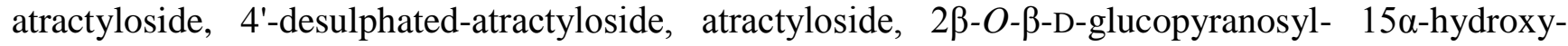
kaur-16-en-18,19-dicarboxylic acid).

The presence of toxic kaurene glycosides prevented the researcher from further exploration of therapeutic potential of the genus Xanthium. However, in a recent study the anti-diabetic actions of individual compounds isolated from $X$. strumarium were screened. Caffeoylquinic acid derivatives and phenolic constituents obtained from the plant had inhibitory effect on aldose reductase, $\alpha$-glucosidase, protein tyrosine phosphatase $1 \beta$, and advanced glycation end products [8]. In the light of these results, the aim of this study was to complete the identification of the chemical constituents from the aerial parts of $X$. spinosum by high-performance liquid chromatography coupled with electrospray ionization tandem mass spectrometry (HPLC-ESI-MS/MS).

\section{Materials and methods \\ Plant material}

The flowering aerial parts of Xanthium spinosum L. were collected at the end of October 2014 from the Botanical Garden belonging to the University of Medicine, Pharmacy, Sciences and Technology "George Emil Palade" from Târgu Mureș, Romania (46³3'16.91"N, 24³5'0.51"E). The plant was identified and the voucher specimen $(\mathrm{GB} / \mathrm{H} / 0162 / 2014)$ is deposited at the Faculty of Pharmacy, Department of Pharmaceutical Botany.

\section{Chemicals and solvents}

The chemical reference substances: 4-caffeoylquinic acid, 1,3-dicaffeoylquinic acid (cynarin), 3,5dicaffeoylquinic acid, caffeic acid, and protocatechuic acid were purchased from Sigma-Aldrich (St. Louis, USA), while xanthatin was bought from ChemFaces (Wuhan, China). These standards were chosen, after the phytochemical exploring of the Xanthium genus. Acetonitrile and methanol were of HPLC super gradient grade (Sigma-Aldrich). Water was purified with Millipore (Billerica, MA, USA) Milli-Q equipment. All other chemicals were of analytical reagent grade.

\section{Extraction}

The powdered herb of X. spinosum $(2 \mathrm{~g})$ was extracted twice with $20 \mathrm{~mL}$ of $8: 2(v / v)$ methanol:water by sonication (Braun Labsonic U, Melsungen, Germany) for $2 \times 5 \mathrm{~min}$. The extracts were separated from the plant powder by centrifugation at $6000 \mathrm{rpm}(2500 \mathrm{~g})$ for $10 \mathrm{~min}$. The two methanolic fractions were combined and evaporated to dryness under reduced pressure (Rotavapor, R200, Büchi, Flawil, Switzerland) below $60^{\circ} \mathrm{C}$ before purification by solid-phase extraction (SPE).

SPE was performed on Supelclean LC-18 micro-columns (500 mg, $3 \mathrm{~mL})$, supplied by Supelco (Bellefonte, PA, USA) using a 12-port vacuum manifold processor (LiChrolut extraction unit; Merck). Samples were dissolved in $80 \%(v / v)$ aqueous methanol and were loaded on to the SPE micro columns previously activated by $5 \mathrm{~mL}$ methanol, $5 \mathrm{~mL}$ water and $2.5 \mathrm{~mL}$ methanol. The collected eluate was completed by a further portion of the extract obtained by loading $2.5 \mathrm{~mL}$ of $80 \%$ methanol on to the SPE column. Finally, the samples obtained were evaporated to dryness and were dissolved in $2 \mathrm{~mL}$ of $80 \%$ methanol.

\section{HPLC-DAD-ESI-MS/MS analysis}

For chromatographic separation and mass spectral analysis, an Agilent 1100 HPLC system (degasser, binary gradient pump, autosampler, column thermostat and diode array detector) was used coupled with an Agilent 6410 Triple Quad LC/MS system equipped with ESI ion source (Agilent Technologies, Palo Alto, CA, USA). 
HPLC separations were achieved on a Zorbax SB-C18, Solvent Saver Plus $(3.5 \mu \mathrm{m}, 80 \AA)$ reversed-phase column (150 mm x $3.0 \mathrm{~mm}$ i.d.; Agilent Technologies, Santa Clara, CA, USA). Mobile phase consisted of $0.3 \%(\mathrm{v} / \mathrm{v})$ aqueous formic acid (A) and methanol supplemented with $0.1 \%(\mathrm{v} / \mathrm{v})$ formic acid (B). The following gradient program was applied: $0.00 \mathrm{~min}, 10 \% \mathrm{~B} ; 15.00 \mathrm{~min}, 67.5 \% \mathrm{~B}$; $24.00 \mathrm{~min}, 67.5 \% \mathrm{~B} ; 25.00 \mathrm{~min}, 100 \% \mathrm{~B} ; 29.00 \mathrm{~min} ; 100 \% \mathrm{~B} ; 32.00 \mathrm{~min}, 10 \% \mathrm{~B}$. The solvent flow rate was $0.4 \mathrm{~mL} / \mathrm{min}$, and column temperature was set at $25^{\circ} \mathrm{C}$. The injection volume was $4 \mu \mathrm{L}$.

Electrospray conditions were as follows: drying gas $\left(\mathrm{N}_{2}\right)$ temperature, $350{ }^{\circ} \mathrm{C}$; flow rate, $9 \mathrm{~L} / \mathrm{min}$; nebulizer pressure, 45 psi $\left(\mathrm{N}_{2}\right)$; fragmentor voltage, $100 \mathrm{~V}$; capillary voltage, $3500 \mathrm{~V}$. High purity nitrogen was used as collision gas, and collision energy was changed between 5 and $60 \mathrm{eV}$ according to differences in molecule structures. Full scan mass spectra were recorded in positive and negative ion mode over an $m / z$ range of 50-1000.

\section{Results and discussions}

The HPLC-DAD-ESI-MS/MS analysis of the aqueous-methanolic extract of the aerial part of Xanthium spinosum revealed the presence of the following unequivocally detected constituents: protocatechuic acid (3), 4- $O$-caffeoylquinic acid (13), caffeic acid (15), 3,5-di- $O$-caffeoylquinic acid (22), and xanthatin (35) (Table 1). Three of the five unequivocally detected compounds are mentioned for the first time in $X$. spinosum: protocatechuic acid, 4- $O$-caffeoylquinic acid, and 3,5-di- $O$ caffeoylquinic acid. Protocatechuic acid and 3,5-di- $O$-caffeoylquinic acid were also detected in the aerial part of $X$. strumarium, with a yield of $25.3 \mathrm{mg}$ and $5.8 \mathrm{mg}$, respectively [8]. According to their study, 3,5-di- $O$-caffeoylquinic acid has an inhibitory effect on $\alpha$-glucosidase (antihyperglycemic activity), while protocatechuic acid presents inhibitory effect on $\mathrm{ABTS}^{+}$radical scavenging activity. Caffeoylquinic acids derivatives are the major active principles in the fruits of $X$. strumarium, and are known for their antioxidant, antibacterial and anti-inflammatory effects [26]. Therefore, further analyses are necessary to determine the content of the detected constituents, and to test their biological effects.

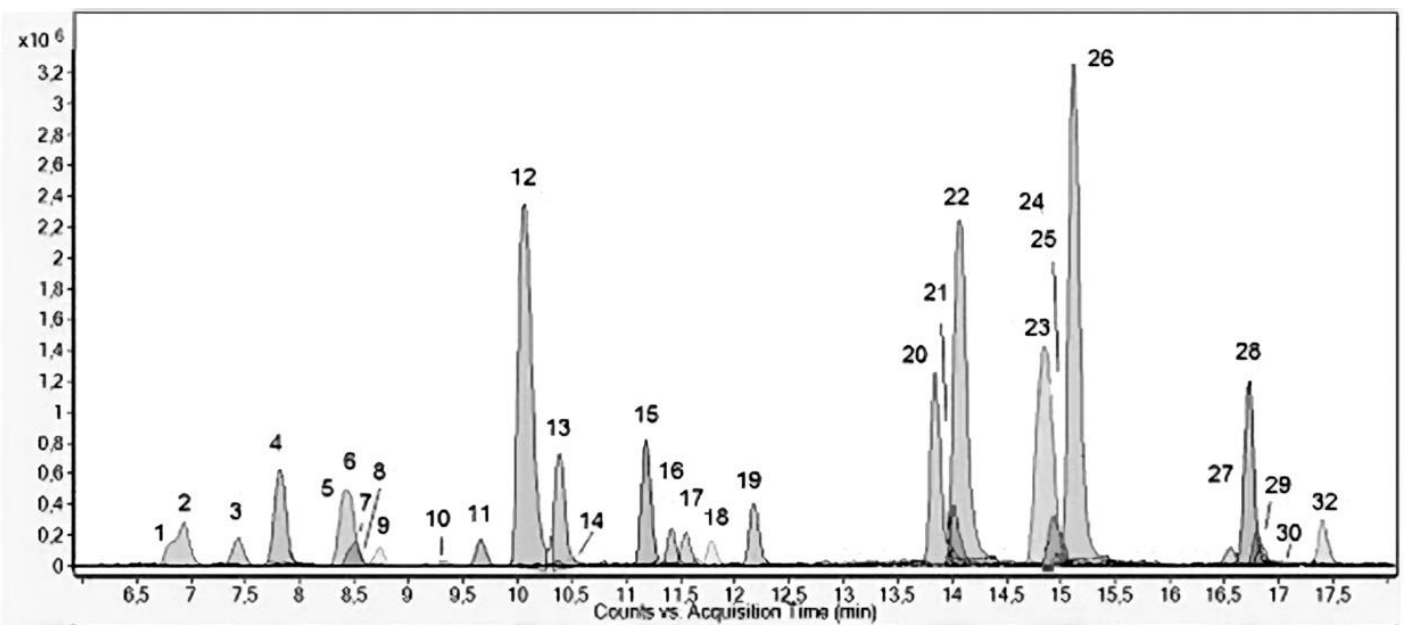

Figure 1. Overlaid extracted ion chromatogram (EIC) acquired in negative ion mode for the aqueous-methanolic extract of Xanthium spinosum aerial part. Peaks refer to Table 1 
Table 1. Peak assignments of compounds detected in aqueous-methanolic extract of Xanthium spinosum aerial part, using fragmentation pattern of the precursor ions and their diagnostic product ions (negative or positive ion mode).

\begin{tabular}{|c|c|c|c|c|c|c|c|}
\hline Peak & Identified compound & $\begin{array}{l}\text { th } \\
(\min )\end{array}$ & $\begin{array}{l}{[\mathrm{M}-\mathrm{H}]^{+}} \\
(m / z)\end{array}$ & $\begin{array}{l}{[\mathrm{M}-\mathrm{H}]^{-}} \\
(m / z)\end{array}$ & $\begin{array}{l}\text { Molecular } \\
\text { formula }\end{array}$ & Fragment ions $(m / z)(R A \%)$ & Reference \\
\hline & Hydroxybenzoic derivatives & & & & & & \\
\hline 1 & Protocatechuic acid- $O$-glucoside (3) & 6.82 & - & 315 & $\mathrm{C}_{13} \mathrm{H}_{16} \mathrm{O}_{9}$ & $\begin{array}{l}315(30), 153(37), 152 \\
(100), 109(21), 108(38)\end{array}$ & Kelebek et al., 2015 [9] \\
\hline 2 & Protocatechuic acid- $O$-glucoside (4) & $6.93^{-}$ & - & 315 & $\mathrm{C}_{13} \mathrm{H}_{16} \mathrm{O}_{9}$ & $\begin{array}{l}315(24), 153(34), 152 \\
(100), 109(13), 108(31)\end{array}$ & Kelebek et al., 2015 \\
\hline 3 & Protocatechuic acid & $7.44^{-1}$ & - & 153 & $\mathrm{C}_{7} \mathrm{H}_{6} \mathrm{O}_{4}$ & $109(100), 108(32), 91(5)$ & Standard \\
\hline 5 & Protocatechuyl-glucose (3) & 8.50 & - & 315 & $\mathrm{C}_{13} \mathrm{H}_{16} \mathrm{O}_{9}$ & $\begin{array}{l}315(5), 153(100), 152(29), \\
109(57)\end{array}$ & Kelebek et al., 2015 \\
\hline 6 & Protocatechuyl-glucose (4) & 8.56 & - & 315 & $\mathrm{C}_{13} \mathrm{H}_{16} \mathrm{O}_{9}$ & $\begin{array}{l}315(27), 153(100), 152 \\
(14), 109(76)\end{array}$ & Kelebek et al., 2015 \\
\hline 7 & Dihydroxybenzoyl-hexose-pentose & 8.58 & - & 447 & $\mathrm{C}_{18} \mathrm{H}_{16} \mathrm{O}_{14}$ & $\begin{array}{l}163(5), 152(100), 109(6) \\
108(32)\end{array}$ & Tahir et al., 2013 [10] \\
\hline 8 & Dihydroxybenzoyl-hexose-pentose & 8.62 & - & 447 & $\mathrm{C}_{18} \mathrm{H}_{16} \mathrm{O}_{14}$ & $\begin{array}{l}163(7), 152(100), 109(15), \\
108(20)\end{array}$ & Tahir et al., 2013 \\
\hline \multirow[t]{2}{*}{11} & $p$-Hydroxybenzoyl-glucose & 9.76 & - & 299 & $\mathrm{C}_{13} \mathrm{H}_{16} \mathrm{O}_{8}$ & $137(100), 93(26)$ & Allen et al., 2015 [11] \\
\hline & Hydroxycinnamic derivatives & & & & & & \\
\hline 4 & 3-O-Caffeoylquinic acid & 7.82 & - & 353 & $\mathrm{C}_{16} \mathrm{H}_{18} \mathrm{O}_{9}$ & $191(100), 179(62), 135(27)$ & Clifford et al., 2003 [12] \\
\hline 9 & $1-O_{-p}$-Coumaroylquinic acid & 8.82 & - & 337 & $\mathrm{C}_{16} \mathrm{H}_{18} \mathrm{O}_{3}$ & $\begin{array}{l}191(100), 163(7), 99(6) \\
93(6)\end{array}$ & Clifford et al., $2007[13$ \\
\hline 10 & 3-O-p-Coumaroylquinic acid & 9.43 & $=$ & 337 & $\mathrm{C}_{6} \mathrm{H}_{18} \mathrm{O}_{3}$ & $191(19), 163(100), 119(12)$ & Clifford et al. 2003 \\
\hline $12-$ & 5-O-Caffeoylquinic acid & 10.07 & - & 353 & $\mathrm{C}_{16} \mathrm{H}_{18} \mathrm{O}_{9}$ & $191(100)$ & Clifford et al., 2003 \\
\hline 13 & 4-O-Caffeoylquinic acid & 10.38 & - & 353 & $\mathrm{C}_{16} \mathrm{H}_{18} \mathrm{O}_{9}$ & $\begin{array}{l}191(56), 179(78), 173 \\
(100), 135(33)\end{array}$ & Standard \\
\hline 14 & 5-O-p-Coumaroylquinic acid & 10.59 & - & 337 & $\mathrm{C}_{16} \mathrm{H}_{18} \mathrm{O}_{3}$ & $191(100)$ & Clifford et al. 2003 \\
\hline 15 & Caffeic acid & 11.18 & - & 179 & $\mathrm{C}_{2} \mathrm{H}_{3} \mathrm{O}_{4}$ & $179(17), 135(100)$ & Standard \\
\hline 16 & cis-5-O-Caffeoylquinic acid & 11.41 & - & 353 & $\mathrm{C}_{16} \mathrm{H}_{18} \mathrm{O}_{9}$ & $191(100)$ & Ncube et al, 2014 [14] \\
\hline 18 & $\begin{array}{l}4-O_{-p} \text {-Coumaroylquinic acid } \\
c i s-5-0-p \text {-Coumaroylquinic acid }\end{array}$ & 11.89 & - & 337 & $\mathrm{C}_{16} \mathrm{H}_{18} \mathrm{O}_{3}$ & $191(100), 173(52), 163(13)$ & Clifford et al., 2003 \\
\hline 19 & 5- $O$-Feruoylquimic acid & 12.17 & - & 367 & $\mathrm{C}_{1} \mathrm{H}_{20} \mathrm{O}_{2}$ & $193(10), 191(100), 173(17)$ & Clifford et al. 2003 \\
\hline 20 & 3,4-di-O-Caffeoylquinic acid & 13.84 & - & 515 & $\mathrm{C}_{25} \mathrm{H}_{24} \mathrm{O}_{12}$ & $\begin{array}{l}515(34), 353(100), 335 \\
(22), 191(26), 179(68), 173 \\
(87), 161(6), 135(5)\end{array}$ & Clifford et al., 2003 \\
\hline 22 & 3,5 -di-O-Caffeoylquinic acid & 14.06 & - & 515 & $\mathrm{C}_{23} \mathrm{H}_{24} \mathrm{O}_{12}$ & $353(91), 191(100), 179(60)$ & Standard \\
\hline 26 & 4,5 -di-O-Caffeoylquinic acid & 15.12 & - & 515 & $\mathrm{C}_{25} \mathrm{H}_{24} \mathrm{O}_{12}$ & $\begin{array}{l}353(100), 191(14), 179 \\
(45), 173(63)\end{array}$ & $\begin{array}{l}\text { Clifford et al., } 2003 \\
\text { Han et al., } 2009[15]\end{array}$ \\
\hline 27 & cis-4,5-di-O-Caffeoylquinic acid & 16.56 & - & 515 & $\mathrm{C}_{25} \mathrm{H}_{24} \mathrm{O}_{12}$ & $\begin{array}{l}353(100), 191(11), 179 \\
(67), 173(52)\end{array}$ & Jaiswal et al., 2010 [16] \\
\hline \multirow[t]{2}{*}{28} & 3,4,5-tri-O-Caffeoylquinic acid & 16.80 & - & 677 & $\mathrm{C}_{34} \mathrm{H}_{30} \mathrm{O}_{15}$ & $\begin{array}{l}677(23), 515(100), \\
353(16)^{2}, \\
335(16), 191(5), 179(75), \\
173(100), 161(9)^{b}\end{array}$ & $\begin{array}{l}\text { Gouveia and Castilho, } \\
2012[17]\end{array}$ \\
\hline & Benayl alcohol derivatives & & & & & & \\
\hline \multirow[t]{2}{*}{17} & $\begin{array}{l}\text { Benzyl alcohol-hexose-pentoge (formate } \\
\text { adduct) }\end{array}$ & 11.54 & - & 447 & - & $\begin{array}{l}401(18), 269(100), 161 \\
(79), 159(7), 101(15)\end{array}$ & Spinola et al., 2014 [18] \\
\hline & Flavonoids & & & & & & \\
\hline 23 & Quercetin-3-O-glucuronide & 14.90 & - & 477 & $\mathrm{C}_{21} \mathrm{H}_{13} \mathrm{O}_{13}$ & $\begin{array}{l}301(94), 283(13), 273(17) \\
255(18), 245(14), 179(58), \\
163(23), 151(100)\end{array}$ & Hwang et al., 2016 [8] \\
\hline 24 & $\begin{array}{l}\text { Quercetin 3-O-(6-O-rhamnosyl-glucoside) } \\
\text { (rutin) }\end{array}$ & 14.99 & - & 609 & $\mathrm{C}_{2} \mathrm{H}_{30} \mathrm{O}_{16}$ & $\begin{array}{l}301(12), 300(100), 271 \\
(10), 151(6)\end{array}$ & Chen et al., 2015 [19] \\
\hline \multirow[t]{2}{*}{25} & Quercetin-3-O-glucoside (isoquercitrin) & 15.04 & - & 463 & $\mathrm{C}_{21} \mathrm{H}_{10} \mathrm{O}_{12}$ & $\begin{array}{l}300(83), 271(100), 255 \\
(59), 179(20), 151(24)\end{array}$ & Liao and $\mathrm{Ku}, 2012$ [20] \\
\hline & Sesquiterpenes & & & & & & \\
\hline 33 & xanthinosin derivative & 17.82 & 249 & - & - & $\begin{array}{l}231(38), 213(8), 207(20), \\
203(36), 189(30), 185(37), \\
175(100), 161(33), 159 \\
(15), 157(58), 145(19), 133 \\
(22)\end{array}$ & Marco et al., 1993 [21] \\
\hline 34 & isoxanthanol & 17.88 & 309 & - & $\mathrm{C}_{17} \mathrm{H}_{34} \mathrm{O}_{3}$ & $\begin{array}{l}291(26), 273(9), 263(6), \\
249(17), 231(11), 213(8), \\
205(16), 203(18), 189(73), \\
185(44), 171(14), 159(18), \\
143(14)\end{array}$ & Marco et al, 1993 \\
\hline 35 & xanthatin & 18.01 & 247 & - & $\mathrm{C}_{15} \mathrm{H}_{18} \mathrm{O}_{3}$ & $\begin{array}{l}229(53), 211(24), 205(43) \\
201(55), 187(75), 183 \\
(100), 159(63), 131(35)\end{array}$ & Standard \\
\hline
\end{tabular}




\begin{tabular}{|c|c|c|c|c|c|c|c|}
\hline 36 & xanthinosin derivative & 18.19 & 249 & - & - & $\begin{array}{l}231(43), 213(21), 203(48), \\
189(24), 187(16), 185(57), \\
175(37), 161(54), 159(85), \\
147(67), 145(90), 133(47), \\
131(47)\end{array}$ & Marco et al, 1993 \\
\hline 37 & 8-epi-xanthatin & 18.51 & 247 & - & $\mathrm{C}_{15} \mathrm{H}_{18} \mathrm{O}_{3}$ & $\begin{array}{l}229(73), 211(24), 205(28), \\
201(68), 187(53), 183(71), \\
173(100), 159(90), 145 \\
(41), 131(64)\end{array}$ & Chen et al., 2013 [22] \\
\hline 38 & 1,5 -epoxy-1,5-dihydroxanthatin & 22.45 & 263 & - & $\mathrm{C}_{15} \mathrm{H}_{13} \mathrm{O}_{4}$ & $\begin{array}{l}235(9), 217(28), 199(17), \\
189(59), 175(12), 161(23), \\
147(36), 133(27), 123(100)\end{array}$ & $\begin{array}{l}\text { Abdei-Mogib et al, } 1991 \\
\text { [23] }\end{array}$ \\
\hline & Diterpenes & & & & & & \\
\hline 21 & 2-O-glucopyranosyl-carboxyatractyligenin & 14.01 & - & 525 & $\mathrm{C}_{26} \mathrm{H}_{33} \mathrm{O}_{14}$ & $\begin{array}{l}481(100), 301(7), 119(13), \\
89(9)\end{array}$ & Lang et al, 2013 [24] \\
\hline 29 & 4 desulphated-carboxyatractyloside & 16,82 & - & 689 & $\mathrm{C}_{11} \mathrm{H}_{45} \mathrm{O}_{15} \mathrm{~S}$ & $\begin{array}{l}645(83), 627(100), 565 \\
(16), 525(8), 343(10), 301 \\
(13), 241(5)\end{array}$ & Carlier et al., 2014 [25] \\
\hline 32 & 3, 4'-didesulphated-carboxyatractyloside & 17,42 & - & 609 & $\mathrm{C}_{31} \mathrm{H}_{46} \mathrm{O}_{12}$ & $\begin{array}{l}565(100), 463(41), 301 \\
(28), 161(8), 143(6), 113 \\
(15), 101(7)\end{array}$ & Carlier et al., 2014 \\
\hline
\end{tabular}

Besides these compounds, 25 phenolics (including 7 hydroxybenzoic derivatives, 13 hydroxycinnamic derivatives, one benzyl alcohol-hexose pentose, and 4 flavonoids), 6 sesquiterpenes and 3 diterpenes were tentatively identified. The detected compounds with the main chromatographic and mass spectrometric data are listed in Table 1. Negative ion mode was used for phenolics and diterpenes (Figure 1), and positive ion mode for sesquiterpenes. A total ion chromatogram (TIC) and a representative extracted ion chromatogram (EIC) of the calibration solution and the plant extract are presented in Figures 2 and 3 for caffeic acid, protocatechuic acid, 4- $O$-caffeoylquinic acid, and 3,5-di$O$-caffeoylquinic acid.
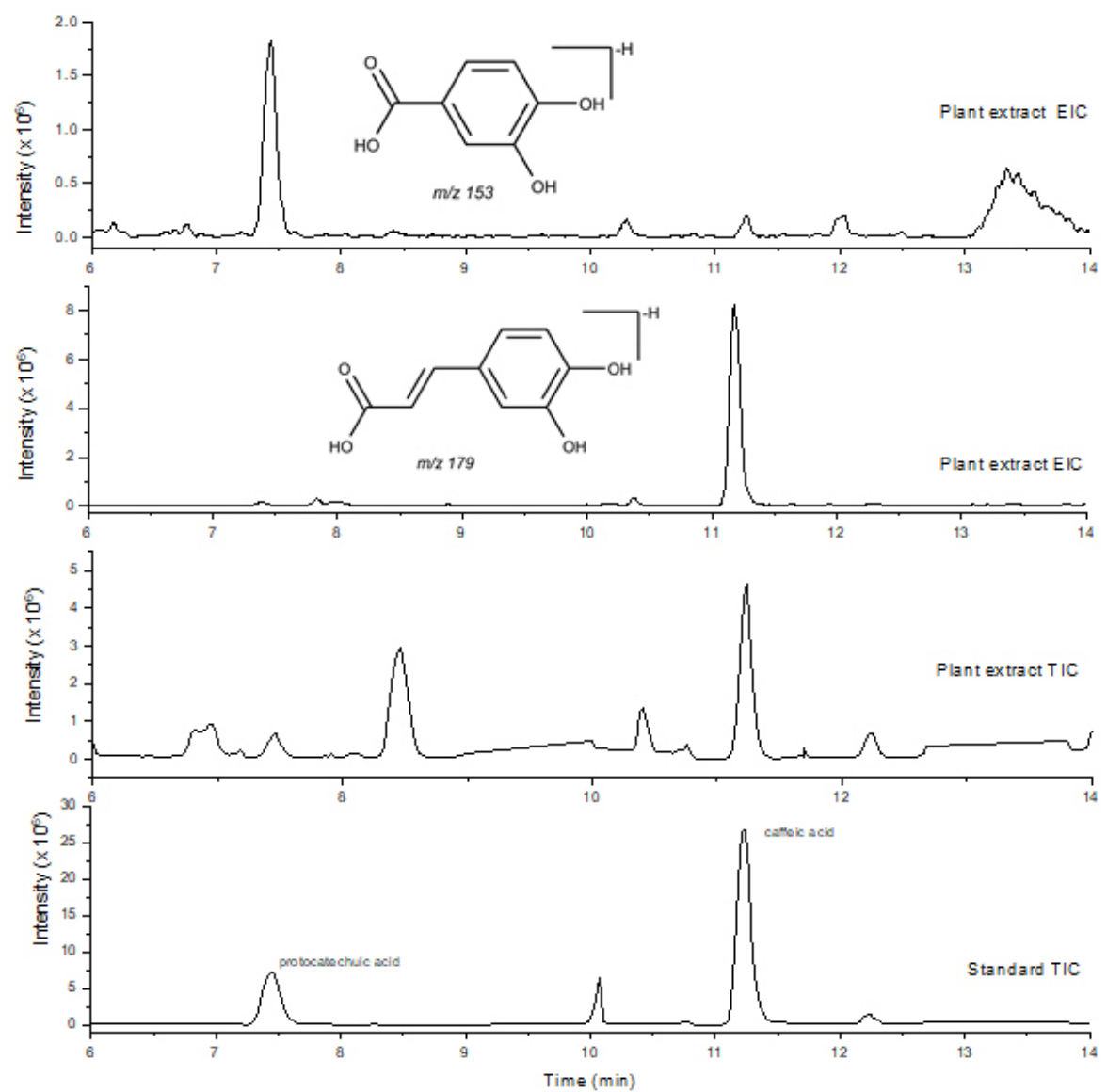

Figure 2. Protocatechuic acid and caffeic acid from the aqueous-methanolic extract of Xanthium spinosum aerial part: total ion chromatogram (TIC) and 
extracted ion chromatogram (EIC) of the calibration solution and the plant extract
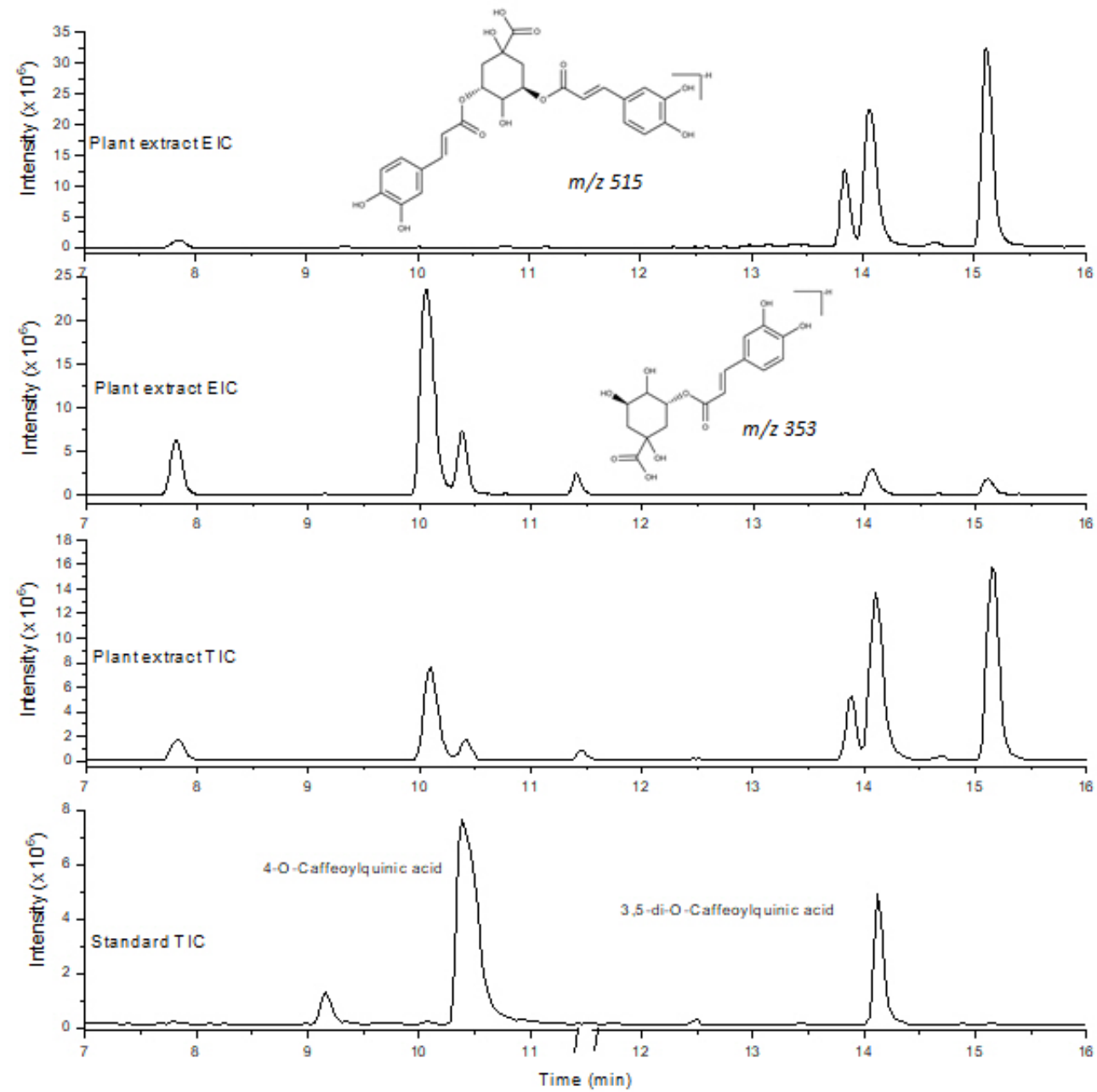

Figure 3. 4- $O$-Caffeoylquinic acid, and 3,5-di- $O$-caffeoylquinic acid from the aqueous-methanolic extract of Xanthium spinosum aerial part: total ion chromatogram (TIC) and extracted ion chromatogram (EIC) of the calibration solution and the plant extract

\section{Conclusions}

HPLC-ESI-MS/MS was applied for the analysis of the metabolite profile of aerial parts of Xanthium spinosum. Protocatechuic acid, 4- $O$-caffeoylquinic acid, and 3,5-di- $O$-caffeoylquinic acid are reported for the first time in $X$. spinosum. Further analyses are necessary to establish the role of the detected compounds in the biological activities, including the anti-inflammatory and antioxidant effects of $X$. spinosum.

Acknowledgements:This work was supported by the Transylvanian Museum Society and Semmelweis University research grant number 129/P.2.EMEOGYSZ, 2014.

\section{References}

1.RACZ, G., RACZ, K.E., SZABO, L.GY., GALENUS KIADO, Budapest, 2012.

2.GINESTA-PERIS, E., GARCIA-BREIJO, F.J., PRIMO-YÚFERA, E., Lett Appl Microbiol, 18, 1994, p. 206-208. 
3.Bader, A., Giner, R.M., Martini, F., Schinella, G.R., Ríos, J.L., Braca, A., Prieto, J.M., Fitoterapia, 91, 2013, p. 284.

4.ROMERO, M., ZANUY, M., ROSELL, E., CASCANTE, M., PIULATS, J., FONT-BARDIA M., BALZARINI J., DE CLERQ E., PUJOL M.D., Eur J Med Chem, 90, 2015, p. 491.

5.PETCU, P., ANDRONESCU, E., Farmacia, 28, no. 4, 1980, p. 217.

6.VARGA, E., MARCU, S.T., ADORYÁN, B., Acta Pharm Hung, 84, 2014, p. 63.

7.DOMOKOS, E., KURSINSZKI, L., KELEMEN, H., VARGA, E., Acta Pharm Hung, 86, 2016, p. 35.

8.HWANG, S.H., WANG, Z., YOON, H.N., LIM, S.S., Molecules, 21, 2016, p. 1241.

9.KELEBEK, H., SELLI, S., GUBBUK, H., GUNES, E., Food Chem, 173, 2015, p. 912.

10.TAHIR, N.I., SHAARI, K., ABAS, F., GHULAM, AHMAD, A.P., HASHIM, A.T., RAMLI, U.S., J Oil Palm Res, 25, 2013, p. 72.

11.ALLEN, F., GREINER, R., WISHART, D., Metabolomics, 11, 2015, p. 98.

12.CLIFFORD, M.N., JOHNSTON, K.L., KNIGHT, S., KUHNERT, N., J Agric Food Chem, 51, 2003, p. 2900.

13.CLIFFORD, M.N., WU, W., KIRKPATRICK, J., KUHNERT, N., J Agric Food Chem, 55, 2007, p. 929.

14.NCUBE, E.N., MHLONGO, M.I., PIATER, L.A., STEENKAMP, P.A., DUBERY, I.A., MADALA, N.E., Chem Cent J, 8, 2014, p. 66.

15.HAN, T., ZHANG, Q.Y., ZHANG, H., WEN, J., WANG, Y., HUANG, B.K., RAHMAN, K., ZHENG, H.C., QIN, L.P., Anal. Chim. Acta., 634, 2009, p. 272.

16.JAISWAL, R., PATRAS, M.A., ERAVUCHIRA, P.J., KUHNERT, N., J Agric Food Chem, 58, 2010 , p. 8722.

17.GOUVEIA, S.C., CASTILHO, P.C., Food Res Int, 45, 2012, p. 362.

18.SPINOLA, V., LLORENT-MARTÍNEZ, E.J., GOUVEIA, S., CASTILHO, P.C., J Agric Food Chem, 62, 2014, p.9722.

19.CHEN, Y., YU, H., WU, H., PAN, Y., WANG, K., JIN, Y., ZHANG, CH., Molecules, 20, 2015, p. 18352.

20.LIAO, CH.-L., KU, K.-L., Anal Chem, 84, 2012, p. 7440.

21.MARCO, J.A., SANZ-CERVERA, J.F., CORRAL, J., CARDA, M., JAKUPOVIC, J., Phytochemistry, 34, 1993, p.1569.

22.CHEN, F., HAO, F., LI, C., GOU, J., LU, D., GONG, F., TANG, H., ZHANG, Y., PLoS ONE, 2, no. 8, 2013, e76621.

23.ABDEI-MOGIB, M., DAWIDAR, A.M., METWALlY, M.A., ABOU-ELZAHAB, M., Phytochemistry, 30, 1991, p. 3461

24.LANG, R., FROMME, T., BEUSCH, A., WAHL, A., KLINGENSPOR, M., HOFMANN, T., Phytochemistry, 93,2013, p. 124.

25.CARLIER, J., ROMEUF, L., GUITTON, J., PRIEZ-BARALlON, C., BEVALOT, F., FANTON, L., GAILLARD, Y., J Anal Toxicol, 38, 2014, p. 619.

26.JIANG, H., YANG, L., XING, X., YAN, M., GUO, X., YANG, B., WANG, Q., KUANG, H., Molecules, 23, no. 2, 2018, pii: E243. doi: 10.3390/molecules23020243.

$\overline{\text { Manuscript received: 6.09.2019 }}$ 
